\title{
Endoscopic management of patients with excessive central airway collapse: a narrative review
}

\author{
Fayez Kheir ${ }^{1}$, Juan Pablo Uribe ${ }^{1 \wedge}$, Sanket Thakore $^{2}$, Adnan Majid ${ }^{1}$ \\ ${ }^{1}$ Division of Thoracic Surgery and Interventional Pulmonology, Beth Israel Deaconess Medical Center, Harvard Medical School, Boston, MA, USA; \\ ${ }^{2}$ Division of Pulmonary, Critical Care and Sleep Medicine, Yale University School of Medicine, New Haven, CT, USA \\ Contributions: (I) Conception and design: F Kheir, JP Uribe; (II) Administrative support: F Kheir, A Majid; (III) Provision of study materials or \\ patients: None; (IV) Collection and assembly of data: F Kheir, JP Uribe, S Thakore; (V) Data analysis and interpretation: F Kheir, JP Uribe, S \\ Thakore; (VI) Manuscript writing: All authors; (VII) Final approval of manuscript: All authors. \\ Correspondence to: Fayez Kheir, MD. Division of Thoracic Surgery and Interventional Pulmonology, Beth Israel Deaconess Medical Center, Harvard \\ Medical School, 185 Pilgrim Road, Deaconess 201, Boston, MA 02215, USA. Email: Fkheir@bidmc.harvard.edu.
}

\begin{abstract}
To provide a narrative review of the novel endoscopic approaches available in the literature for the treatment of excessive central airway collapse (ECAC). ECAC is characterized by excessive expiratory collapse of the central airway which can lead to dyspnea, cough, difficulty clearing secretions and recurrent respiratory infections. Surgical stabilization of the airway via tracheobronchoplasty (TBP) has been considered the standard of care for patients who have severe symptomatic ECAC despite optimal medical management. However, a significant proportion of patients are not adequate candidates for surgery. We performed a systematic search of MEDLINE/PubMed and ClinicalTrials.gov databases, then narrative reviews of identified reports, focusing on those published in the last 10 years. Of 129 references to ECAC from a MEDLINE/PubMed search, 54 were from the last 10 years and 30 were the focus of this review. Further cross reference was made to ClinicalTrials.gov for 1 study, however the results have not yet been published and the study is currently underway. Endoscopic thermo-ablative techniques such as argon plasma coagulation (APC) has been evaluated in ex vivo as well as in vivo models, showing promising results; However human studies are underway. Laser TBP and custom designed stents are currently under development, although the evidence is limited to case reports and case series. Novel endoscopic treatments are promising for the treatment of ECAC. However, more studies are needed to prove efficacy.
\end{abstract}

Keywords: Excessive central airway collapse (ECAC); tracheobronchomalacia (TBM); tracheobronchoplasty (TBP); bronchoscopy

Received: 28 January 2021; Accepted: 10 June 2021; Published: 20 April 2022.

doi: 10.21037 /jovs-21-5

View this article at: https://dx.doi.org/10.21037/jovs-21-5

\section{Introduction}

\section{Background}

Excessive central airway collapse (ECAC) is characterized by expiratory excessive tracheal and/or mainstem bronchial collapse which can lead to dyspnea, cough, difficulty clearing mucous and recurrent respiratory infections $(1,2)$. ECAC comprises of two entities: tracheobronchomalacia
(TBM) and excessive dynamic airway collapse (EDAC). TBM is characterized by weakening of airway wall cartilages and softening of anterolateral tracheobronchial wall. During exhalation, it leads to cartilaginous wall collapse and excessive narrowing of the airway diameter (1-3). EDAC is characterized by atrophy of the muscular fibers in the posterior membrane of the airway wall without affecting cartilaginous structure of anterolateral wall. During

$\wedge$ ORCID: 0000-0002-5122-3613. 
exhalation, the posterior membranous wall bulges anteriorly and causes excessive narrowing of the airway diameter without cartilaginous wall collapse (1-3). The reported prevalence of ECAC in the literature varies between $4.1 \%$ to $23.3 \%$, mainly because of non-specific symptoms and inconsistencies in diagnostic criteria $(1,2,4-6)$. The clinical characteristics and diagnostic workup are similar for both $\mathrm{TBM}$ and EDAC with subtle differences in non-surgical therapeutic approach (1-7). The treatment of ECAC is mainly centered on improving symptoms and quality of life; however, this might not be achieved with medical therapy alone and some patients require surgical stabilization of the posterior membrane [tracheobronchoplasty (TBP)]. Furthermore, a significant number of patients are not amenable to undergo surgery leaving a gap in the treatment strategy. In the following review we discuss the new endoscopic modalities available for the treatment of ECAC. These modalities might be the only option to improve symptoms in patients not amenable to undergo surgery. We present the following article in accordance with the Narrative Review reporting checklist (available at https:// jovs.amegroups.com/article/view/10.21037/jovs-21-5/rc).

\section{Objectives}

We aim to review and discuss the novel endoscopic approaches available in the literature for the treatment of ECAC.

\section{Methods}

A literature search of MEDLINE and PubMed was performed by the authors and revealed a total of 129 publications with the search term "Excessive Central Airway Collapse" (January-2021). When the search was narrowed to the last 10 years, 54 articles were obtained. An Additional Search in ClinicalTrials.gov showed another study, however the results have not yet been published and the study is currently underway. Finally, a total of 30 articles were selected through full-text review and included in the manuscript.

\section{Discussion}

The goals of therapy in patients with ECAC is improving symptoms and quality of life. Hence, treatment should initially focus on optimizing comorbidities that are frequently associated with ECAC such as asthma, chronic obstructive pulmonary disease (COPD), vocal cords dysfunction, gastroesophageal reflux disease (GERD), sleep apnea, obesity and decondition $(1,2,7,8)$. Additionally, patients with difficulties clearing mucous secretions that might lead to recurrent lower respiratory tract infections, should be trained on pulmonary hygiene techniques such as breathing exercises, flutter valve, percussion vest and postural drainage $(2,8)$.

Majid et al. (9), in a cohort of 139 patients with ECAC, found that $63(45.3 \%)$ had documented GERD as shown by an abnormal esophageal $\mathrm{pH}$ test result. When such patients $(n=63)$ were treated aggressively with medical therapy or anti-reflux surgery, 46\% (23 subjects) reported improvement in respiratory symptoms and did not require further treatment for ECAC. Furthermore, inadequately treated GERD can negatively impact the outcomes of surgical TBP (2,8-10).

Continuous positive airway pressure (CPAP) has demonstrated to improve symptoms such as dyspnea, cough, mucous clearance, and exercise tolerance in patients with ECAC. When applied in affected patients, it works as a pneumatic stent that improves central airways patency during expiration $(2,11,12)$. CPAP is usually considered as a "bridge" to more definitive surgical TBP; however, if patients with ECAC are not surgical candidates, or do not tolerate long term airway stent, CPAP can be offered as a long-term therapy. In addition, some patients may have residual symptoms following TBP and they can benefit from long-term CPAP $(7,11,13)$. CPAP titration under direct bronchoscopic visualization has been suggested to determine adequate CPAP pressure that will keep central airways patent. Currently, a randomized control trial including patients with Mounier-Kuhn syndrome or TBM is ongoing to evaluate the optimal pressure titration via bronchoscopy (ClinicalTrails.gov NCT03101059) (14). CPAP of 7 to $10 \mathrm{cmH}_{2} \mathrm{O}$ has been reported to be usually adequate $(11,15,16)$. Additionally, in patients who are severely deconditioned, pulmonary rehabilitation with CPAP should be considered before surgery or as maintenance therapy. Currently, studies are being carried out to determine the impact of CPAP on exercise capacity (17).

Patients who fail to respond to aggressive medical therapy might benefit from surgical stabilization of airways with TBP. Surgery was traditionally performed through a right thoracotomy and was associated with somerespiratory-morbidity and mortality. It is currently being offered through a minimally invasive approach using the robotic platform. We will discuss both approaches in 
this series, but regardless, a thorough evaluation of who will benefit from TBP is paramount to ensure optimal outcomes. A short-term stent trial for 7 to 14 days is the only method practiced by most centers to establish which patients will benefit from TBP $(1,2,18)$.

\section{Stenting for ECAC}

\section{Short-term stent trial}

Patients with severe ECAC who fail to respond to medical therapy and have significant symptoms affecting their quality of life, are evaluated by thoracic surgery. If patients are deemed to be good surgical candidates, they undergo short-term stent trial.

Silicone stents have been historically used as a stent trial for 2 weeks to assess whether patients symptoms and quality of life improve and thus decide if they will benefit from surgical airway stabilization $(1,2,19,20)$. However, silicone stents are also associated with high complication rate such as mucous plug, granulation tissue, infection, stent migration and intractable coughing. When patients develop such symptoms during stent trial, it can be difficult to determine if symptoms are from stent related complication or failure of stent trial $(2,19)$.

Uncovered self-expanding metallic airway stents (USEMAS) have been suggested as an alternative to silicone stents for short term stent trials. Advantages of USEMAS over silicone stents for short term stent trial are (I) easy to deploy, (II) preferable inner diameter to wall thickness ratio, (III) better ability to conform to tortuous airways, (IV) preserved innate muco-ciliary apparatus which helps with mucous clearance.

Majid et al. (8) reported a retrospective review of 33 patients with severe symptomatic ECAC who underwent short stent trial with USEMAS. A total of 25 (75.6\%) patients had responded positively to the stent trial based on combination of improvement in self-reported respiratory symptoms, objective measurements, and functional status, which compares favorably to $55 \%$ positive response with silicone stents reported previously $(8,19,20)$. The serious complications of USEMAS (defined as stent migration, obstruction, respiratory failure, hemoptysis, pneumothorax) were reported to be $6.1 \%$, which also compares favorably to previously reported silicone stent complication rate before $(40 \%)$ and after (9\%) adoption of a protocol using mucolytic and expectorant therapy $(19,21)$. The study reported that removal of USEMAS was easy without significant granulation tissue.

Our group had an accepted manuscript for publication comparing the use of silicone Y-stents (SYSs) and USEMAS during the stent trial before TBP. A total of 60 patients were included with severe ECAC; 42 (70\%) underwent USEMAS placement, while 18 patients (30\%) had an SYS placed. After propensity score matching, the SYS group showed an increased rate of mucus plugging $(38.5 \%$ vs. $0 \%, \mathrm{P} \leq 0.047)$. Although not statically significant, but a clinical improvement was observed in modified Medical Research Council (mMRC) scale, Cough Quality of Life Questionnaire (CQLQ), and 6-minute walk test (6MWT) in the USEMAS group during the stent trial (22).

Some centers are forgoing short-term stent trial $(7,23)$ and patients are directly referred to TBP if severe symptoms match the imaging and bronchoscopic finding of severe expiratory airway collapse. However, our group recommend a short-term stent trial before subjecting patients to TBP. Our unpublished experience demonstrated that $75 \%$ of patients with severe ECAC who are appropriate surgical candidates undergo stent trial will experience subjective and/or objective improvement, and $65 \%$ will ultimately undergo TBP.

\section{Long-term stents for ECAC}

Acute respiratory failure requiring mechanical ventilation due to ECAC can occur and usually manifests as failure to liberate patients from mechanical ventilation due to the loss of positive pressure keeping the airway lumen patent (2). Silicone-Y or dynamic stent placement might be considered in such patients who are not amenable to immediate surgical intervention with TBP. Murgu et al. (24) described a case of a 74-year-old man with obesity and obstructive sleep apnea with recurrent episodes of respiratory failure secondary to ECAC. CPAP failed due to the patient's subjective intolerance and lack of improvement. Silicone stents were placed in the left main bronchus, right main bronchus and distal trachea (total of three silicone stents), and the patient was eventually weaned off from mechanical ventilation. It is important to mention that these patients require a close clinical follow-up and adequate muco-ciliary clearance techniques to minimize the risk of mucous plugging and infection. Frequently these patients are treated with mucolytics, oral oscillatory device such as flutter valves, and airway humidification with nebulized hypertonic saline and acetylcysteine (25). 


\section{Customized three-dimensional (3D) printed stents}

It has been more than three decades since the first commercial $3 \mathrm{D}$ printer was available in the market and accessible to the public, facilitating its implementation in modern medicine (26). This technology has a potentially major role in interventional pulmonology, especially in the management of complex airway disease, tailoring the treatment strategy to a patient-specific anatomical approach in the pediatric as well as adult population (27).

The airways can be compared to fingerprints and tracheobronchial diseases unique to each patient. Furthermore, the airway has dynamic motion with inspiration, expiration, and cough, which varies in every patient. Precision medicine focuses on optimizing efficacy and therapeutic benefit for a particular patient, thus improving outcomes $(28,29)$. The cornerstone of this technology relies on the use CT data and bronchoscopic images to build stents that can be matched to a particular anatomical situation, applying the optimized expansion force and achieving an almost perfect alignment with the mucosa. These factors may decrease the typical stentrelated complications such as biofilm formation, mucus retention between the airway wall and the stent, formation of granulation tissue, and stent migration (27).

Schweiger et al. (28) reported a case of a 69-year-old patient with severe TBM that initially underwent TBP with posterior mesh stabilization of the trachea and both main bronchi 4 years before, with only temporary relief of symptoms. The patient was reevaluated with dynamic bronchoscopy and dynamic CT, and due to the dimensions of the central airway (largest diameter $32 \mathrm{~mm}$ ), conventional stents were not considered. A patient-specific SYS was designed and manufactured to obtain the optimal size match and placed via bronchoscopy. After placement, the alignment with the mucosal lining was appropriate. The patient was followed for 8 months showing sustained relief of symptoms to his highest satisfaction, allowing him to return to his normal daily activities without restrictions. The same group also presented a similar case of a 71-year-old patient with severe TBM that had undergone TBP with initial improvement, however with subsequent clinical deterioration a year after the surgery (28). This patient underwent placement of a 15 -mm dynamic Y-stent (not 3D-customized stent), and despite an imperfect fit of the anteroposterior diameter and length, he reported subjective improvement. Therefore, a decision was made to offer the patient a customized Y-stent, resulting in appropriate alignment with the airways. After 5 months of follow-up, the patient showed consistent improvement of symptoms. Both patients receive periodic follow-up bronchoscopies, with stent cleansing every 4 to 6 weeks. Mucus clearance was supported by inhalation therapy.

\section{Bronchoscopic thermoablative techniques}

\section{Argon plasma coagulation (APC)}

Surgical stabilization of the airway with TBP is still considered as the most definitive treatment modality for patients with severe symptomatic ECAC following optimal medical management. Buitrago et al. (30) described a cohort of 161 patients that underwent TBP and found that the surgery improved quality of life, exercise capacity, as well as respiratory symptoms. However, they reported a 30-day mortality of $1.2 \%$ and post-operative complications in $47 \%$ of patients.

Thermoablative therapy such as APC, might play an important role with favorable safety profile and likely less health care resource utilization. APC can cause tissue devascularization and induction of fibrosis, thus potentially decreasing the movement and collapsibility of the posterior tracheal wall during inspiration in patients with EDAC (31).

de Lima et al. (32) described the effect of four thermoablative techniques at different power settings [electrocautery, radiofrequency ablation (RFA), potassium titanyl phosphate (KTP) laser, and precise APC] in the tracheobronchial tree of four sheep cadavers. They described the histologic changes consistent with acute thermal injury defined as epithelium ablation, elastin and collagen fiber condensation, smooth muscle cell cytoplasm condensation with hyper/eosinophilic change, and finally chondrocyte nuclei pyknosis. Treatment effect was observed with all techniques in all tissue compartments layers to varying degrees. Electrocautery and RFA using low power settings (defined as $10 \mathrm{~W}$ ) showed isolated thermal injury with potentially viable tissue across all histologic layers. However, at higher power settings (15-20 W), they described the same thermal injury but associated with partial or complete tissue erosion, leaving no chances for tissue regeneration. Regarding KTP laser, they observed isolated thermal injury at $20-30 \mathrm{~W}$ with complete tissue erosion and no potential regeneration of the epithelium exclusively. When they increased the energy of KTP laser to $40 \mathrm{~W}$, complete tissue erosion was noted at the epithelium along with thermal injury over the remaining layers. Finally, when precise APC was used, independently of the effect or power 


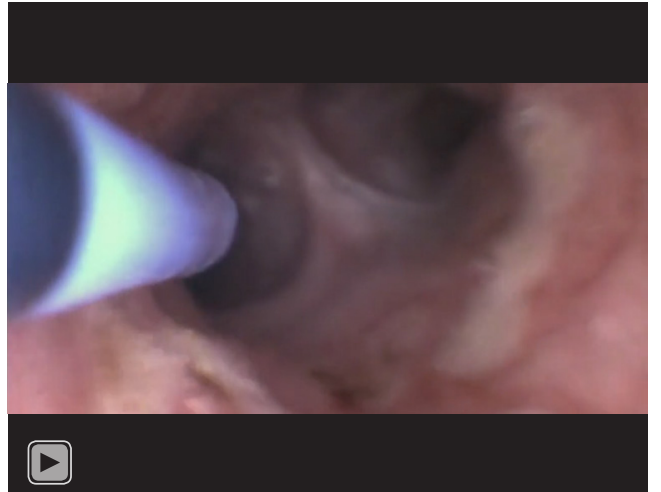

Video 1 Argon plasma coagulation in the posterior membrane of the airway in an animal model.

setting (effect 3-5-7), they observed a consistently more pronounced thermal injury across all tissue compartment layers when compared with the other modalities, without erosion thus preserving potentially viable tissue in all layers. Of all the four modalities, APC was the only technique able to achieve consistent and homogeneous thermal injury across all tissue layers with no evidence of complete erosion and leaving a potential for tissue regeneration and fibrosis. The main limitation of this study was that all techniques were evaluated in an ex vivo model (tissues are non-perfused with no tissue regenerative capacity).

Recently, Kheir et al. (33) evaluated the effect of bronchoscopic APC under precise settings (effects or power setting 3-5-7 and for 3-5 seconds) in the posterior membrane of adult live sheep (see Video 1). They were able to follow the animals for 30 days, with a CT scan at day 2 and bronchoscopy at day 30 before euthanasia. Subsequently, they described the histopathologic changes along with the expression of genes (TGFB1, cFOS1, cFOS2, $c$ - fun and $I F N-\gamma)$ associated with fibrosis using Quantitative Reverse Transcription-PCR from the treated sheep, and then compared them with tracheal specimens from 2 untreated sheep (control). They reported no significant clinical events during the 30-day period of follow up, with no need for reinterventions. CT chest imaging on day 2 did not show any signs of pneumomediastinum or pneumothorax. Bronchoscopy on day 30 did not show any stenotic segments, perforations, or evidence of infection. The tracheobronchial tree of both animals showed an adequate healing process with complete reconstitution of the epithelium within the treated area (Figure 1). Regarding the histopathologic findings, there were only minor surface epithelial changes and the submucosal smooth muscle layer remained intact as compared to controls; However, more drastic changes were seen in the deeper aspects of the posterior membrane underlying the smooth muscle. They found a significant increase in the qualitative density and amount of fibroelastic collagen deposition in the treated areas. Compared with the controls, where the fibrous layer of the posterior membrane measured $\sim 700 \pm 70 \mu \mathrm{m}$ on average, the thickness of the fibrous layer for effect 3 averaged $530 \pm 250 \mu \mathrm{m}$, effect 5 averaged $700 \pm 190 \mu \mathrm{m}$, and effect 7 averaged $1,270 \pm 250 \mu \mathrm{m}$. Furthermore, APC treatment increased the expression of cFOS1 as well as c-Jun. There were no changes in the expression of other fibrosis-associated genes such as TGF $\beta 1, \mathrm{cFOS} 2$, and IFN- $\gamma$. Limitations of this study included the small sample size, the use of unrelated untreated controls and the lack of animal models for EDAC; however, they were able to show that APC with high effect increased collagen deposition and the expression of fibrosis-associated genes in the posterior membrane of the tracheobronchial tree without signs of complete erosion or other complications. Human studies are needed to evaluate bronchoscopic APC for patients with severe symptomatic EDAC who are not surgical candidates for TBP.

\section{Laser TBP}

Laser is another thermoablative modality that has been studied in patients with ECAC. This technique produces relatively deep furrows into the submucosa while still preserving areas of intact mucosa between layers. This is felt to increase wound healing while still promoting scarring to stiffen the mucosa, decreasing the movement and collapsibility of the posterior tracheal wall during inspiration. Unfortunately, the current evidence available in the literature is limited to a case report and a case series. Almost decade ago, Dutau et al. (34) described a patient with TBM due to Mounier-Kuhn syndrome who was treated with Yttrium Aluminium Perovskite (YAP) laser on the posterior membrane. They reported a significant clinical improvement after the procedure, and bronchoscopy 6 weeks later revealed marked improvement of the collapsibility of the airways. A second treatment was performed 14 months later due to slight recurrence of the posterior membrane collapse; however, the patient had remained clinically asymptomatic for almost a decade having required a total of four treatments with YAP laser. Subsequently, Castellanos et al. (35) in 2017 published 

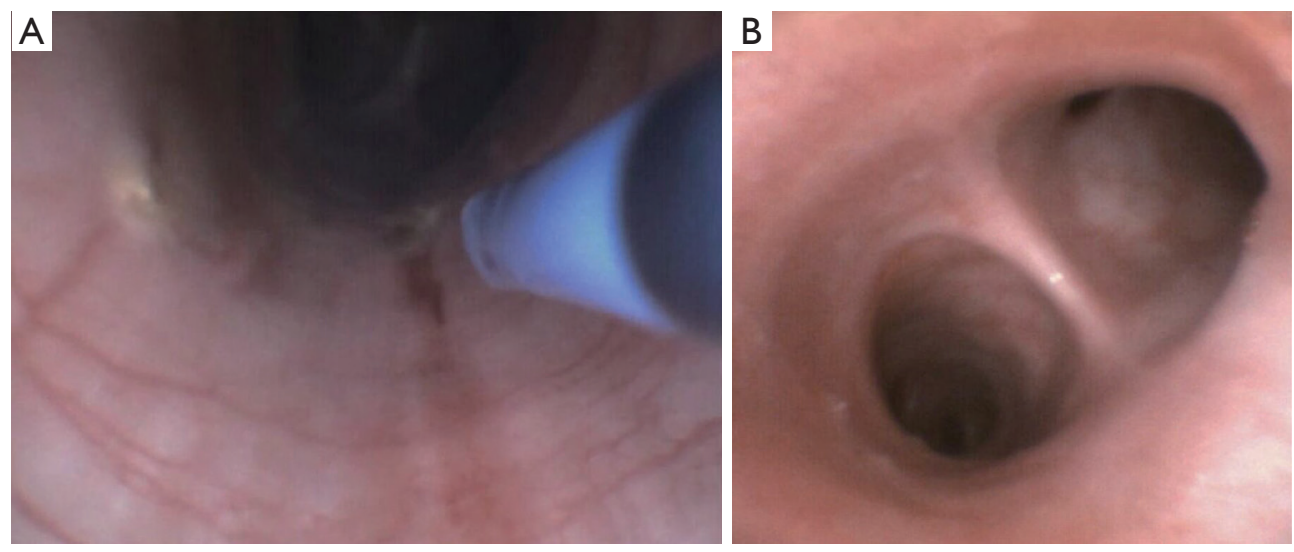

Figure 1 Bronchoscopy APC. (A) APC in the posterior membrane of the airway in an animal model. (B) Bronchoscopic follow up 30 days later after the procedure revealing adequate mucosal healing and no signs of stenosis or perforations. APC, argon plasma coagulation.

a case series of 10 patients diagnosed with TBM, most likely EDAC, defined as airway collapsibility greater than $50 \%$ during exhalation. These patients were treated with two to three holmium laser scarring interventions via bronchoscopy (mean number of procedures 2.3-energy delivered 1,600 J). The laser was used to strafe the mucosa of the posterior membrane within the tracheobronchial tree longitudinally, transversely, and in a serpentine manner from distal to proximal. Patients were followed up to 6 months, with no intra or post-operative mortality. All subjects showed subjective improvement in their respiratory symptoms, with no complications during the follow up period.

\section{Summary}

ECAC is characterized by expiratory excessive tracheal and /or mainstem bronchial collapse which can lead to dyspnea, cough, difficulty clearing mucous and recurrent respiratory infections, and this condition may overlap with other more common respiratory conditions such as asthma and COPD. Treatment is centered on aggressive medical management of the comorbidities, pulmonary hygiene, non-invasive ventilation and pulmonary rehabilitation. Stent trial is a reliable way to identify which patients might benefit from surgical central airway stabilization (TBP); however, when the patients are not adequate surgical candidates, aggressive medical management used to be the only option. Currently, new endoscopic approaches such as thermoablative techniques and custom designed stents are being studied and developed for the treatment of patients with EDAC and
TBM respectively. These new techniques seem promising, especially for patients who are not surgical candidates; however, large human data is needed to establish the safety and long-term efficacy of bronchoscopic management of ECAC.

\section{Acknowledgments}

Funding: None.

\section{Footnote}

Provenance and Peer Review: This article was commissioned by the Guest Editors (Charles T. Bakhos and Abbas E. Abbas) for the series "Tracheobronchoplasty" published in Fournal of Visualized Surgery. The article has undergone external peer review.

Reporting Checklist: The authors have completed the Narrative Review reporting checklist. Available at https:// jovs.amegroups.com/article/view/10.21037/jovs-21-5/rc

Peer Review File: Available at https://jovs.amegroups.com/ article/view/10.21037/jovs-21-5/prf

Conflicts of Interest: All authors have completed the ICMJE uniform disclosure form (available at https://jovs. amegroups.com/article/view/10.21037/jovs-21-5/coif). The series "Tracheobronchoplasty" was commissioned by the editorial office without any funding or sponsorship. The authors have no other conflicts of interest to declare. 
Ethical Statement: The authors are accountable for all aspects of the work in ensuring that questions related to the accuracy or integrity of any part of the work are appropriately investigated and resolved.

Open Access Statement: This is an Open Access article distributed in accordance with the Creative Commons Attribution-NonCommercial-NoDerivs 4.0 International License (CC BY-NC-ND 4.0), which permits the noncommercial replication and distribution of the article with the strict proviso that no changes or edits are made and the original work is properly cited (including links to both the formal publication through the relevant DOI and the license). See: https://creativecommons.org/licenses/by-nc-nd/4.0/.

\section{References}

1. Murgu S, Colt H. Tracheobronchomalacia and excessive dynamic airway collapse. Clin Chest Med 2013;34:527-55.

2. Kheir F, Majid A. Tracheobronchomalacia and excessive dynamic airway collapse: medical and surgical treatment. Semin Respir Crit Care Med 2018;39:667-73.

3. Hammond K, Ghori UK, Musani AI. Tracheobronchomalacia and excessive dynamic airway collapse. Clin Chest Med 2018;39:223-8.

4. Ridge CA, O'donnell CR, Lee EY, et al. Tracheobronchomalacia: current concepts and controversies. J Thorac Imaging 2011;26:278-89.

5. Carden KA, Boiselle PM, Waltz DA, et al. Tracheomalacia and tracheobronchomalacia in children and adults: an indepth review. Chest 2005;127:984-1005.

6. Ikeda S, Hanawa T, Konishi T, et al. Diagnosis, incidence, clinicopathology and surgical treatment of acquired tracheobronchomalacia. Nihon Kyobu Shikkan Gakkai Zasshi 1992;30:1028-35.

7. McGinn J, Herbert B, Maloney A, et al. Quality of life outcomes in tracheobronchomalacia surgery. J Thorac Dis 2020;12:6925-30.

8. Majid A, Alape D, Kheir F, et al. Short-term use of uncovered self-expanding metallic airway stents for severe expiratory central airway collapse. Respiration 2016;92:389-96.

9. Majid A, Kheir F, Alape D, et al. The prevalence of gastroesophageal reflux in patients with excessive central airway collapse. Chest 2019;155:540-5.

10. Gangadharan SP, Bakhos CT, Majid A, et al. Technical aspects and outcomes of tracheobronchoplasty for severe tracheobronchomalacia. Ann Thorac Surg 2011;91:1574-
80; discussion 1580-1.

11. Adliff M, Ngato D, Keshavjee S, et al. Treatment of diffuse tracheomalacia secondary to relapsing polychondritis with continuous positive airway pressure. Chest 1997;112:1701-4.

12. Ferguson GT, Benoist J. Nasal continuous positive airway pressure in the treatment of tracheobronchomalacia. Am Rev Respir Dis 1993;147:457-61.

13. Tamura Y, Sakatani K, Yamakoshi N, et al. A case of severe COPD associated with tracheo-bronchial stenosis, treated with non-invasive positive pressure ventilation. Nihon Kokyuki Gakkai Zasshi 2008;46:915-20.

14. Airway collapse in patients with Mounier-Kuhn syndrome: titration with positive pressure to reduce collapse. ClinicalTrials.gov. [cited 2021 Mar 23]. Available online: https://clinicaltrials.gov/ct2/show/NCT03101059

15. Wiseman NE, Duncan PG, Cameron CB. Management of tracheobronchomalacia with continuous positive airway pressure. J Pediatr Surg 1985;20:489-93.

16. Murgu SD. Pneumatic stenting for tracheobronchomalacia. J Bronchology Interv Pulmonol 2014;21:109-12.

17. Effect of CPAP on 6-minute walk test outcomes in patients with ECAC. ClinicalTrials.gov. [cited 2021 Jan 27]. Available online: https://clinicaltrials.gov/ct2/show/ NCT04217603

18. López-Padilla D, García-Luján R, Puente Maestu L, et al. Tracheobronchomalacia treatment: how far have we come? J Thorac Dis 2016;8:3490-3.

19. Ernst A, Majid A, Feller-Kopman D, et al. Airway stabilization with silicone stents for treating adult tracheobronchomalacia: a prospective observational study. Chest 2007;132:609-16.

20. Majid A, Guerrero J, Gangadharan S, et al. Tracheobronchoplasty for severe tracheobronchomalacia: a prospective outcome analysis. Chest 2008;134:801-7.

21. Odell DD, Majid A, Gangadharan SP, et al. Adoption of a standardized protocol decreases serious complications of airway stenting in patients with tracheobronchomalacia. Chest 2010;138:784A.

22. Alape D, Kheir F, Gangadharan S, et al. Stent trial for expiratory central airway collapse: does the type of stent really matter? Chest 2017;152:A53.

23. Wright CD, Mathisen DJ. Tracheobronchoplasty for tracheomalacia. Ann Cardiothorac Surg 2018;7:261-5.

24. Murgu SD, Cherrison LJ, Colt HG. Respiratory failure due to expiratory central airway collapse. Respir Care 2007;52:752-4.

25. Parikh M, Wilson J, Majid A, et al. Airway stenting in 
excessive central airway collapse. J Vis Surg 2017;3:172.

26. Freitag L, Gördes M, Zarogoulidis P, et al. Towards individualized tracheobronchial stents: technical, practical and legal considerations. Respiration 2017;94:442-56.

27. Alraiyes AH, Avasarala SK, Machuzak MS, et al. 3D printing for airway disease. AME Med J 2019;4:14.

28. Schweiger T, Gildea TR, Prosch H, et al. Patientspecific, 3-dimensionally engineered silicone Y-stents in tracheobronchomalacia: Clinical experience with a novel type of airway stent. J Thorac Cardiovasc Surg 2018;156:2019-21.

29. Morrison RJ, Hollister SJ, Niedner MF, et al. Mitigation of tracheobronchomalacia with 3D-printed personalized medical devices in pediatric patients. Sci Transl Med 2015;7:285ra64. Erratum in: Sci Transl Med 2015;7:287er4.

30. Buitrago DH, Majid A, Alape DE, et al. Singlecenter experience of tracheobronchoplasty for tracheobronchomalacia: perioperative outcomes. Ann Thorac Surg 2018;106:909-15.

31. Reichle G, Freitag L, Kullmann HJ, et al. Argon plasma

doi: 10.21037/jovs-21-5

Cite this article as: Kheir F, Uribe JP, Thakore S, Majid A. Endoscopic management of patients with excessive central airway collapse: a narrative review. J Vis Surg 2022;8:14. coagulation in bronchology: a new method--alternative or complementary? Pneumologie 2000;54:508-16.

32. de Lima A, Vidal B, Kheir F, et al. Effects of electromechanical techniques over sheep tracheal tissue: an ex-vivo pilot study. In: C75. Lung measurement: novel methods and techniques. American Thoracic Society, 2018:A5852.

33. Kheir F, Ospina-Delgado D, Beattie J, et al. Argon plasma coagulation (APC) for the treatment of excessive dynamic airway collapse (EDAC): an animal pilot study. J Bronchology Interv Pulmonol 2021. [Epub ahead of print]. doi: 10.1097/LBR.0000000000000746.

34. Dutau H, Maldonado F, Breen DP, et al. Endoscopic successful management of tracheobronchomalacia with laser: apropos of a Mounier-Kuhn syndrome. Eur J Cardiothorac Surg 2011;39:e186-8.

35. Castellanos P, Mk M, Atallah I. Laser tracheobronchoplasty: a novel technique for the treatment of symptomatic tracheobronchomalacia. Eur Arch Otorhinolaryngol 2017;274:1601-7. 